\title{
A New Approach to the Stabilization of the Wave Equation with Boundary Damping Control
}

\author{
Boumediène Chentouf and Mohamed S. Boudellioua
}

Department of Mathematics and Statistics, College of Science, Sultan Qaboos University, P.O. Box 36, Al Khod 123, Muscat, Sultanate of Oman, Email: chentouf@squ.edu.om..
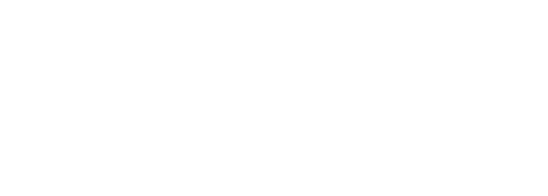

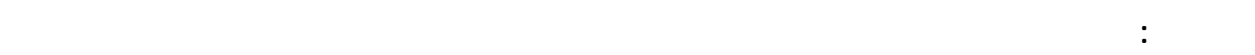

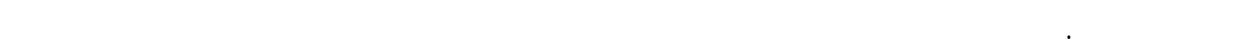

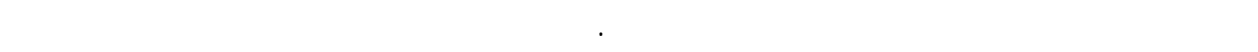
يتعلق بلالثروط الأسلسية فتط.

\begin{abstract}
This paper deals with boundary feedback stabilization of a system, which consists of a wave equation in a bounded domain of $\mathbb{R}^{n}$, with Neumann boundary conditions. To stabilize the system, we propose a boundary feedback law involving only a damping term. Then using a new energy function, we show that the solutions of the system asymptotically converge to a stationary position, which depends on the initial data. Similar results were announced without proof in (Chentouf and Boudellioua, 2004).
\end{abstract}

KEYWORDS: Wave equation, Neumann condition, boundary damping control, energy function, asymptotic behavior.

\section{Introduction}

T et $\Omega$ be a bounded open connected set in $\mathbb{R}^{n}$ having a smooth boundary $\Gamma=\partial \Omega$ of class $C^{2}$. Liven a partition $\left(\Gamma_{0}, \Gamma_{1}\right)$ of $\Gamma$, consider the following wave equation:

$$
y_{t t}(x, t)-\Delta y(x, t)=0, \quad \text { in } \Omega \times(0, \infty)
$$

with Neumann boundary conditions and initial conditions 


$$
\begin{cases}\frac{\partial y}{\partial v}(x, t)=0, & \text { on } \Gamma_{0} \times(0, \infty) \\ \frac{\partial y}{\partial v}(x, t)=U(t), & \text { on } \Gamma_{1} \times(0, \infty) \\ y(x, 0)=y_{0}(x), y_{t}(x, 0)=z_{0}(x), & \end{cases}
$$

where $v$ is the unit normal of $\Gamma$ pointing towards the exterior of $\Omega$ and $U$ is a feedback law to be determined. Note that $\Gamma_{1}$ is supposed to be nonempty whereas $\Gamma_{0}$ may be empty. that is,

As in Chentouf and Boudellioua (2004), we propose a feedback depending only on a damping term,

$$
U(t)=-a(x) y_{t}(x, t), \quad(x, t) \in \Gamma_{1} \times(0, \infty)
$$

where $a \in L^{\infty}\left(\Gamma_{1}\right)$ satisfies $a(x) \geq a_{0}>0$ for any $x \in \Gamma_{1}$. Then, it is proved that the solutions of the closed loop system asymptotically tend towards a constant depending on the initial data $y_{0}$ and $z_{0}$.

There is a rich literature concerning the stabilization problem of the wave equation (see for instance Bardos et al. (1992), Chen (1979a, 1979b, 1981a, 1981b), Komornik (1994), Lax et al. (1963), Lagnese (1988), Lions (1988a, 1988b), Morawetz (1975), Quinn and Russell (1977), Triggiani (1975) and the references therein). In all references cited above, at least one of the following conditions is assumed to be satisfied:

- The equation (1.1) involves also the displacement term $y$.

- The stabilizing feedback law $U(t)$ contains not only a boundary dissipation term $y_{t}$ but also the boundary displacement $y$.

- The first boundary condition in (1.2) involves the displacement term $y$ (the boundary condition (1.2) is replaced for instance by $y=0$ or $\frac{\partial y}{\partial v}+y=0$ on $\Gamma_{0} \times(0, \infty)$.

The main contribution of this paper is to provide an alternative proof of Lagnese's result (1983) by means of a simple and direct method. The key idea of the proof is to introduce a new energy norm (see Chentouf and Boudellioua (2004) for similar systems and Conrad et al. (2002) for one-dimensional wave equation).

\section{Preliminaries and Well-posedness of the Problem}

In this section, we study the existence and uniqueness of the solutions of the closed-loop system (1.1)(1.3). Assume, without loss of generality, that for any $x \in \Gamma_{1}, a(x)=a$, where $a$ is a positive constant. Then, consider the state space

$$
H=H^{1}(\Omega) \times L^{2}(\Omega),
$$

equipped with the inner product 


$$
\begin{aligned}
\langle(y, z)(\tilde{y}, \tilde{z})\rangle_{H} & =\int_{\Omega}(\nabla y \nabla \tilde{y}+z \tilde{z}) d x \\
& +\varepsilon\left(\int_{\Omega} z d x+\int_{\Gamma_{1}} a y d \sigma\right)\left(\int_{\Omega} \tilde{z} d x+\int_{\Gamma_{1}} a \tilde{y} d \sigma\right),
\end{aligned}
$$

where $\varepsilon>0$ is a constant to be determined.

The first result is stated in the following proposition:

Proposition 1. The state space $H=H^{1}(\Omega) \times L^{2}(\Omega)$ endowed with the inner product (2.1) is a Hilbert space provided that $\varepsilon$ is small enough.

Proof of Proposition 1. It suffices to show that the norm $\|\cdot\|_{H}$ induced by the inner product (2.1) is equivalent to the usual one $\|\cdot\|_{H^{1}(\Omega) \times L^{2}(\Omega)}$, that is, the existence of two positive constants $K$ and $\tilde{K}$ such that

$$
K\|(y, z)\|_{H^{1}(\Omega) \times L^{2}(\Omega)} \leq\|(y, z)\|_{H} \leq \tilde{K}\|(y, z)\|_{H^{1}(\Omega) \times L^{2}(\Omega)} .
$$

Applying Holder's inequality and using trace Theorem Adams (1976) (see also Mikhaïlov (1980)) yield:

$$
\begin{aligned}
\|(y, z)\|_{H}^{2} & \leq \int_{\Omega}\left(|\nabla y|^{2}+[1+2 \varepsilon \operatorname{mes}(\Omega)]|z|^{2}\right) d x \\
& +2 \varepsilon a^{2} C_{1} \operatorname{mes}\left(\Gamma_{1}\right)\left(\int_{\Omega}\left(|\nabla y|^{2}+(1+2 \varepsilon)|y|^{2}\right) d x\right),
\end{aligned}
$$

where $C_{1}$ is a positive constant depending on $\Omega$ (see Adams (1976) or Mikhaïlov (1980)). Therefore (2.2) holds for a positive constant $\tilde{K}$ depending on $\varepsilon$, a, mes $\left(\Gamma_{1}\right)$ and $m e s(\Omega)$.

For the reverse inequality, we proceed as follows:

$$
\begin{aligned}
\|(y, z)\|_{H}^{2} & =\int_{\Omega}\left(|\nabla y|^{2}+|z|^{2}\right) d x+\varepsilon\left(\int_{\Omega} z d x\right)^{2}+\varepsilon\left(\int_{\Gamma_{1}} a y d \sigma\right)^{2} \\
& +2 \varepsilon\left(\int_{\Gamma_{1}} a y d \sigma\right)\left(\int_{\Omega} z d x\right) .
\end{aligned}
$$

Obviously for any $\delta>0$, Young's inequality yields

$$
2\left(\int_{\Gamma_{1}} a y d \sigma\right)\left(\int_{\Omega} z d x\right) \geq-a \delta^{-1}\left(\int_{\Omega} z d x\right)^{2}-a \delta\left(\int_{\Gamma_{1}} y d \sigma\right)^{2}
$$

Combining (2.4) and (2.3), we get

$$
\|(y, z)\|_{H}^{2} \geq \int_{\Omega}\left(|\nabla y|^{2}+|z|^{2}\right) d x+\varepsilon\left(1-a \delta^{-1}\right)\left(\int_{\Omega} z d x\right)^{2}+\varepsilon a(a-\delta)\left(\int_{\Gamma_{1}} y d \sigma\right)^{2} .
$$

Furthermore, using a classical compactness argument, one can show the following generalized Poincare inequality:

$$
\int_{\Omega}|y|^{2} d x \leq C_{2}\left\{\int_{\Omega}|\nabla y|^{2} d x+\left(\int_{\Gamma_{1}} y d \sigma\right)^{2}\right\},
$$

where $C_{2}>0$ depends on $\Omega$. This, together with (2.5), implies that 


$$
\begin{aligned}
\|(y, z)\|_{H}^{2} & \geq \int_{\Omega}\left\{\left[\varepsilon a(a-\delta) C_{2}^{-1}\right]|y|^{2}+[1-\varepsilon a(a-\delta)]|\nabla y|^{2}+|z|^{2}\right\} d x \\
& +\varepsilon(\delta-a) \delta^{-1}\left(\int_{\Omega} z d x\right)^{2},
\end{aligned}
$$

for any $\delta<a$. Finally, applying again Holder's inequality to the last term in (2.6), one can show the existence of a positive constant $K$ depending on $a, \delta$ and mes $(\Omega)$ such that the second inequality in (2.2) holds, provided that $\delta<a$ and $\varepsilon$ satisfies the following condition

$$
\varepsilon<\min \left(\frac{1}{a(a-\delta)}, \frac{\delta}{(a-\delta) \operatorname{mes}(\Omega)}\right) .
$$

This concludes the proof of Proposition 1.

We turn now to the formulation of the closed-loop system (1.1)-(1.3) in an abstract form on $H$. Let $z(\cdot, t)=y_{t}(\cdot, t)$ and $\Phi(t)=(y(\cdot, t), z(\cdot, t))$. Then, the closed loop system can be written as follows

$$
\left\{\begin{array}{l}
\Phi_{t}(t)=A \Phi(t), \\
\Phi(0)=\Phi_{0}=\left(y_{0}, z_{0}\right),
\end{array}\right.
$$

where $A$ is an unbounded linear operator defined by

$$
D(A)=\left\{\begin{array}{c}
(y, z) \in H^{1}(\Omega) \times H^{1}(\Omega) ; \Delta y \in L^{2}(\Omega) ; \\
\frac{\partial y}{\partial v}=0 \text { on } \Gamma_{0} ; \frac{\partial y}{\partial v}+a z=0 \text { on } \Gamma_{1}
\end{array}\right\},
$$

and for any $(y, z) \in D(A)$,

$$
A(y, z)=(z, \Delta y) \text {. }
$$

It is a simple task to check that the operator $-A$, defined by (2.8)-(2.9), is maximal monotone. Therefore, it follows from semigroups theory Pazy (1983) (see also Brezis (1992)) that:

Lemma 1. (i) The linear operator $A$ generates a $C_{0}$ semigroup of contractions $S(t)$ on $H=\overline{D(A)}$. (ii) For any initial data $\Phi_{0}=\left(y_{0}, z_{0}\right) \in D(A)$, the system (2.7) has a unique strong solution $\Phi(t)=\left(y(\cdot, t), y_{t}(\cdot, t)\right)=S(t) \Phi_{0} \in D(A)$ for all $t \geq 0$ such that $\Phi(\cdot)=\left(y, y_{t}\right) \in$ $C^{1}\left(\mathbb{R}^{+} ; H\right) \cap C\left(\mathbb{R}^{+} ; D(A)\right)$. Moreover, the function $t \mapsto\|A \Phi(t)\|_{H}$ is decreasing.

(iii) For any initial data $\Phi_{0}=\left(y_{0}, z_{0}\right) \in H$, the system (2.7) has a unique weak solution $\Phi(t)=\left(y(\cdot, t), y_{t}(\cdot, t)\right)=S(t) \Phi_{0} \in H$ such that $\Phi(\cdot)=\left(y, y_{t}\right) \in C^{0}\left(\mathbb{R}^{+} ; H\right)$. 


\section{Asymptotic Behavior}

In this section, we will show an asymptotic behavior result for the unique solution of (2.7) in the state space $H=H^{1}(\Omega) \times L^{2}(\Omega)$. To do so, we shall first show the following lemma:

Lemma 2. The resolvent operator $(\lambda I-A)^{-1}: H \rightarrow H$ is compact for any $\lambda>0$ and hence the canonical embedding $i: D(A) \rightarrow H$ is compact, where is equipped with the graph norm.

Proof of Lemma 2. Let $(f, g,) \in H$. We seek $(y, z) \in D(A)$ such that $(I-A)(y, z)=(f, g)$. Equivalently,

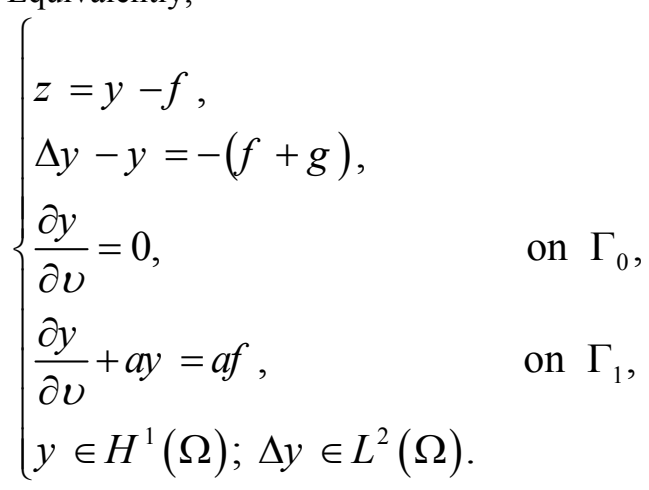

Using Lax-Milgram Theorem (see Brezis (1992)), one can readily show that the above system has a unique solution and thus the operator $(I-A)^{-1}$ exists and maps $H$ into $D(A)$. Finally, by using Sobolev embedding, we deduce that $(I-A)^{-1}$ is compact. The proof of Lemma 2 follows then from the wellknown result of Kato (1976).

The main result of this paper is:

Theorem 1. For any initial data, $\Phi_{0}=\left(y_{0}, z_{0}\right) \in H$, the solution $\Phi(t)=\left(y(\cdot, t), y_{t}(\cdot, t)\right)$ of (2.7) tends in $H$ to $(C, 0)$ as $t \rightarrow+\infty$, where

$$
C=\left\{\operatorname{ames}\left(\Gamma_{1}\right)\right\}^{-1}\left(\int_{\Omega} z_{0} d x+\int_{\Gamma_{1}} a y_{0} d \sigma\right) .
$$

Proof of Theorem 1. By a standard argument of density of $D\left(A^{2}\right)$ in $H$ and the contraction of the semigroup $S(t)$, it suffices to prove Theorem 1 for smooth initial data $\Phi_{0}=\left(y_{0}, z_{0}\right) \in D\left(A^{2}\right)$. Let $\Phi(t)=\left(y(\cdot, t), y_{t}(\cdot, t)\right)=S(t) \Phi_{0}$ be the solution of (2.7). It follows from (ii) of Lemma 1 that the trajectory of solution $\{\Phi(t)\}_{t \geq 0}$ is a bounded set for the graph norm and thus precompact in $H$ by virtue 
of Lemma 2. Applying LaSalle's principle, we deduce that $\omega\left(\Phi_{0}\right)$ is non-empty, compact, invariant under the semigroup $S(t)$ and $S(t) \Phi_{0} \rightarrow \omega\left(\Phi_{0}\right)$ as $t \rightarrow \infty[10]$. In order to prove the strong stability, it suffices to show that $\omega\left(\Phi_{0}\right)$ reduces to $(C, 0)$. To this end, let $\tilde{\Phi}_{0}=\left(\tilde{y}_{0}, \tilde{z_{0}}\right) \in \omega\left(\Phi_{0}\right) \subset D(A)$ and $\tilde{\Phi}(t)=\left(\tilde{y}(\cdot, t), \tilde{y}_{t}(\cdot, t)\right)=S(t) \tilde{\Phi}_{0} \in D(A)$ the unique strong solution of (2.7). Recall that it is well-known that $\|\tilde{\Phi}(t)\|_{H}$ is constant Haraux (1991) and thus $\frac{d}{d t}\left(\|\tilde{\Phi}(t)\|_{H}^{2}\right)=0$, i.e.,

$$
<A \tilde{\Phi}, \tilde{\Phi}>_{H}=-\int_{\Gamma_{1}} a|z|^{2} d \sigma=0
$$

This implies that $\tilde{z}=\tilde{y_{t}}=0$ on $\Gamma_{1}$ and therefore $\tilde{y}$ is solution of the system System:

$$
\begin{cases}\tilde{y}_{t t}-\Delta \tilde{y}=0, & \\ \frac{\partial y}{\partial v}=0, & \text { on } \Gamma_{0}, \\ \tilde{y}_{t}=\frac{\partial \tilde{y}}{\partial v}=0, & \text { on } \Gamma_{1}, \\ \tilde{y}(0)=\tilde{y}_{0} ; \tilde{y}_{t}=\tilde{z}_{0}, & \\ \tilde{y} \in H^{1}(\Omega) ; \Delta \tilde{y} \in L^{2}(\Omega), & \end{cases}
$$

whereas $\tilde{z}=\tilde{y}_{t}$ is solution of the system

$$
\begin{cases}\tilde{z_{t t}}-\Delta \tilde{z}=0 & \\ \frac{\partial \tilde{z}}{\partial v}=0, & \text { on } \Gamma_{0}, \\ \tilde{z}=\frac{\partial \tilde{z}}{\partial v}=0, & \text { on } \Gamma_{1}, \\ \tilde{z}(0)=\tilde{z_{0}} ; \tilde{z_{t}}(0)=\Delta \tilde{y}_{0} . & \end{cases}
$$

Obviously, to deduce the desired result, it suffices to show that $\tilde{y}=$ constant is the only solution of (3.2). To do so, we first use the standard Holmgren's uniqueness theorem (see John (1982)) for the system (3.3) to conclude that $\tilde{z} \equiv 0$. Thus the system (3.2) is reduced to the elliptic problem:

$$
\left\{\begin{array}{l}
\Delta \tilde{y}=0, \\
\frac{\partial \tilde{y}}{\partial v}=0, \text { on } \Gamma,
\end{array}\right.
$$

which clearly yields that $\tilde{y} \equiv$ constant. This, together with (3.1), implies the desired result. 
Remark 1. Integrating with respect to $x$ and $t$ and using Green formula for the closed loop system (1.1)(1.3), we obtain the following identity:

$$
\int_{\Omega} y_{t} d x+\int_{\Gamma_{1}} a y d \sigma=\int_{\Omega} z_{0} d x+\int_{\Gamma_{1}} a y_{0} d \sigma .
$$

Furthermore, if the initial data $\left(y_{0}, z_{0}\right)$ satisfies the additional condition

$$
\int_{\Omega} z_{0} d x+\int_{\Gamma_{1}} a y{ }_{0} d \sigma=0
$$

then the constant $C$ of Theorem 1 is zero, i.e., $\left(y(\cdot, t), y_{t}(\cdot, t)\right) \rightarrow(0,0)$ in $H$ as $t \rightarrow+\infty$.

\section{Acknowledgements}

The authors acknowledge the support of Sultan Qaboos University (Grant IG/SCI/DOMS/02/08). The authors are also grateful to the referees for their useful remarks and helpful suggestions.

\section{References}

ADAMS, R.A. 1975. Sobolev spaces. Pure and Applied Mathematics, vol. 65. Academic Press, New YorkLondon.

BARDOS, C., LEBAU, G., and RAUCH, J. 1992. Sharp sufficient conditions for the observation, controllability and stabilization of waves from the boundary. SIAM J. Control Optim., 30(5): 10241064.

BREZIS, H. 1992. Analyse Fonctionnelle, Théorie et Applications. Paris: Masson.

CHEN, G. 1979a. Energy decay estimates and exact boundary value controllability for the wave equation in a bounded domain. J. Math. Pures Appl., 58: 249-274.

CHEN, G. 1979b. Control and stabilization for the wave equation in a bounded domain. Part I, SIAM J. Control Optim. 17: 66-81.

CHEN, G. 1981a. Control and stabilization for the wave equation in a bounded domain. Part II, SIAM J. Control Optim., 19: 114-122.

CHEN, G. 1981b. A note on boundary stabilization of the wave equations. SIAM J. Control Optim., 19: 106-113.

CHENTOUF, B. and BOUDELLIOUA, M.S. 2004. On the stabilization of the wave equation with dynamical control. In: Proc. 16th International Symposium on Mathematical Theory of Networks and Systems, 2004, Leuven, Belgium, 6 pages.

CONRAD, F., O'DOWD, G. and SAOURI, F.Z. 2002. Asymptotic behaviour for a model of flexible cable with tip masses. Asymptot. Anal., 30: 313-330.

HARAUX, A. 1991. Systèmes Dynamiques Dissipatifs et Applications. Paris.

JOHN, F. 1982. Partial Differential Equations. (2nd Edition) Springer-Verlag.

KATO, T. 1976. Perturbation theory of linear Operators. Springer-Verlag.

KOMORNIK, V. 1994. Exact Controllability and Stabilization. The Multiplier Method. Masson, Paris.

LAX, P.D, MORAWETZ, C.S, and PHILIPS, R.S. 1993. Exponential decay of solutions of the wave equation in the exterior of a star-shaped obstacle. Comm. Pure Appl. Math., 65: 447-486.

LAGNESE, J. 1983. Decay of solutions of the wave equation in a bounded region with boundary dissipation. J. Diff. Equ., 50: 163-182.

LAGNESE, J. 1988. Note on boundary stabilization of wave equations. SIAM J. Control Optim., 26(5): $1250-1256$. 
LIONS, J.L. 1988a. Exact controllability, stabilization and perturbations for distributed systems. SIAM Review, 30(1): 1-68.

LIONS, J.L. 1988b. Contrôlabilité exacte et stabilisation de systèmes distribués. 1, Masson, Paris.

MAJDA, A. 1975. Disappearing solutions for the dissipative wave equations. Indiana Univ. Math. J., 24: 1119-1133.

MIKHAÏLOV, V. 1980. Equations aux dérivées partielles. Mir, Moscou 1980.

MORAWETZ, C.S. 1975. Decay of solutions of the exterior problem for the wave equations. Comm. Pure Appl. Math., 28: 229-264.

PAZY, A. 1983. Semigroups of Linear Operators and Applications to Partial Differential Equations. Springer-Verlag, New York.

QUINN, J.P., and RUSSELL, D.L. 1977. Asymptotic stability and energy decay rate for solutions of hyperbolic equations with boundary damping. Proc. Roy. Soc. Edinburgh. 77: 97-127.

RAUCH, J., and TAYLOR, M. 1974. Exponential decay of solutions to hyperbolic equations in bounded domain. Indiana. Univ. Math. J., 24(1): 79-86.

RUSSELL, D.L. 1978. Controllability and stabilizability theory for linear partial differential equations: Recent progress and open questions. SIAM Review, 20(4): 639-739.

TRIGGIANI, R. 1989. Wave equation on a bounded domain with boundary dissipation: an operator approach. J. Math. Anal. Appli., 137: 438-461.

Received 28 February 2004

Accepted 18 December 2004 